\title{
ZUM VERHÄLTNIS VON HEIMATVERLUST UND NEUBEGINN IN FRÜHEN ROMANEN ÜBER FLUCHT UND VERTREIBUNG
}

\begin{abstract}
On the relation between loss and new start in early novels on flight and expulsion
This article looks at early West German novels that depict flight and expulsion of Germans after World War II. It is already in the 1950s that German literature takes up on East German home loss; especially authors originating from the Eastern parts make flight and expulsion subject of their works. How then do those novels reflect the radical change - as a beginning, as an ending? What did 1945 mean to those Germans who had to flee their homes beyond Oder and Neisse? - The end of German culture in Eastern Europe? The final surrender of their homelands? And how could the expellees restart their life in the Western parts? The article interlaces these questions with the texts' narrative time structures. Most of the novels focus on the process of flight and expulsion and skip the arrival and integration in the West. Thus, the novels attest the uncertainty inherent in the new beginning. The article argues that the authors do not narrate the ending of the transition because in the $1950 \mathrm{~s}$ for them it has not ended yet. It investigates three representative novels: Engel, Menschen und Dämonen (1951) by Hanna Stephan, Die schlesische Barmherzigkeit (1950) by Ruth Hoffmann and Ein Abschied (1951) by Karl Borree.
\end{abstract}

\section{Problemaufriss}

1945: Ende der nationalsozialistischen Diktatur - Verheißung eines Neubeginns. Was aber bedeutete das Jahr für den Teil des deutschen Volkes, der ab 1945 seine Heimat hinter Oder und Neiße verlassen musste? Das Ende der deutschen Kultur in Osteuropa? Die endgültige Aufgabe der Heimat? Und wie konnte es für die Flüchtlinge und Vertriebenen einen Neubeginn im Westen geben?

Im Folgenden soll untersucht werden, auf welche Weise exemplarisch gewählte Fiktionen der 1950er Jahre den Heimatverlust verarbeiten und erzählerisch inszenieren. Grundsätzlich konstituieren sich narrative Texte aus Handlungen und Ereignissen, die in einer temporalen Verbindung stehen. Anfang und Ende und somit Transformationen organisieren jedes Narrativ: „Was in einer Geschichte letztlich erzählt wird, ist der Veränderungsprozess, wie es vom Ausgangszustand zum Endzustand 
kommt." "Welchen Anfang (welche Anfänge) und welches Ende (welche Enden) erzählen die Texte über Flucht und Vertreibung? Wie reflektieren diese Texte das Jahr 1945? Wie verhalten sich hier Interpretationen von Anfang und Ende zueinander? Diese Fragen sollen im Folgenden mit Beobachtungen zum erzählerischen Umgang mit Zeit und in den Texten artikulierter Zeitsymbolik verschränkt werden.

\section{Untersuchungsgegenstand: Frühe Erzählungen des ostdeutschen Heimatverlusts}

Bereits in den 1950er Jahren widmet sich die deutsche Literatur dem ostdeutschen Heimatverlust. Es sind insbesondere Autoren mit entsprechendem biografischen Hintergrund (z.B. Ruth Hoffmann, Ernst Wiechert, Kurt Ihlenfeld, Hanna Stephan), die früh über Flucht und Vertreibung schreiben, heute aber zunehmend in Vergessenheit geraten sind. ${ }^{2}$ In der frühen Bearbeitungsphase des Themas fällt eine Mehrzahl von Romanen auf, die sich über eine kurze Erzählzeit erstrecken und das unmittelbare Fluchtgeschehen erzählen. Romane über die Ankunft im Westen und die Integration der Flüchtlinge sind rar. ${ }^{3}$ Die Texte zeugen damit von der Ungewissheit, die dem Neubeginn innewohnt. Sie erzählen, so eine Hypothese, den Übergang nicht zu Ende, weil er für die Autoren Anfang der 1950er nicht zu Ende ist. ${ }^{4}$ Das lässt sich etwa an Ruth Hoffmanns Die schlesische Barmherzigkeit (1950), Karl F. Borées Ein Abschied (1951) und Ruth Storms Das vorletzte Gericht (1953) zeigen. ${ }^{5}$

Alle drei Autoren verfügen über einen biografischen Bezug zu den Orten, von deren Verlust sie erzählen. Ruth Storm kam 1905 in Kattowitz zur Welt, wo sie bis zum Abstimmungsjahr 1921 lebte. ${ }^{6}$ Nachdem Kattowitz polnisch geworden war, zog

\footnotetext{
${ }^{1}$ Karolina Frenzel u.a.: Storytelling: Das Praxisbuch, München/Wien 2006, S. 82. In diesem Sinne behält die von Aristoteles in seiner Poetik vorgenommene triadische Strukturierung von Narrativität in Anfang, Mitte und Ende definitorischen Charakter.

${ }^{2}$ In gegenwärtigen Handbüchern deutscher Literatur tauchen diese Autoren in der Regel nicht mehr bzw. nur kaum auf.

${ }^{3}$ Erst in jüngster Zeit stellen Romane über den ostdeutschen Heimatverlust zunehmend die Ankunft bzw. Integration der Flüchtlinge und Vertriebenen im Westen ins Zentrum, z.B. Reinhard Jirgls Die Unvollendeten (2003) und Christoph Heins Landnahme (2005).

${ }^{4}$ Auch Monika Melchert bemerkt, dass (Berliner) Nachkriegsromane zum Thema in der Regel Beschreibung eines „Interregnums, eines Zustandes des Unentschiedenseins“ seien. Monika Melchert: Die Zeitgeschichtsprosa nach 1945 im Kontext der Schuldfrage, in: Deutsche Erinnerung. Berliner Beiträge zur Prosa der Nachkriegsjahre (1945-1960), hrsg. von Ursula Heukenkamp, Berlin 2000, S. 101-166, hier S. 154.

${ }^{5}$ Ruth Storm: Das vorletzte Gericht, München 1953 (im Folgenden im laufenden Text mit Sigle VG zitiert). Karl Friedrich Borée: Ein Abschied, Wiesbaden 1951 (im Folgenden mit Sigle A zitiert). Ruth Hoffmann: Die schlesische Barmherzigkeit, Köln 1953 (im Folgenden mit Sigle SB zitiert).

${ }^{6}$ Nach dem Ersten Weltkrieg verfügten die Franzosen, dass Deutschland Oberschlesien an Polen abtreten sollte. Es kam zu deutschen Massenprotesten und damit zu einer Volksabstimmung, in deren
} 
Storm ins Riesengebirge. Von 1926 bis 1943 lebte sie in Berlin, kehrte dann aber nach Mittelschreiberhau im Riesengebirge zurück. Beim Einfall der Sowjets 1945 beschloss Storm, nicht zu fliehen, sondern bis zur Vertreibung im Juni 1946 zu bleiben. ${ }^{7}$ Karl F. Borée wurde 1886 in Görlitz geboren. ${ }^{8}$ Zudem war er von 1920 bis 1924 in Königsberg tätig, das Schauplatz der Handlung in Ein Abschied ist. Seit 1924 arbeitete Borée in Berlin, er hat also keine eigenen Erinnerungen an Flucht oder Vertreibung. ${ }^{9}$ Auch Ruth Hoffmann hat Flucht und Vertreibung nicht erlebt. Sie wurde 1893 in Breslau geboren, zog aber 1929 nach Berlin, wo sie fortan als Schriftstellerin tätig war. ${ }^{10}$ Teilen Borée und Hoffmann so nicht die Vertreibungserfahrungen Ruth Storms, eint die drei Schriftsteller doch eine gemeinsame Erfahrung: die des Heimatverlusts. Denn auch für die 1945/46 bereits seit Jahren im Westen lebenden Autoren besiegelten die Potsdamer Beschlüsse diesen Verlust. Sie machten eine Rückkehr in die Landschaften der Kindheit und Erinnerung unmöglich.

Ruth Hoffmanns Die Schlesische Barmherzigkeit (1950) erzählt das Leben von Emma, einer aus ärmlichen Verhältnissen stammenden aufrichtigen und fleißigen Frau. Sie ist zunächst als Wirtschafterin in Breslau bei der Familie Hildebrandt tätig. Insbesondere zu den Kindern Suse und Hans pflegt sie eine innige, liebevolle Beziehung. Nach ihrer Heirat zieht sie in ein Dorf in die Nähe der Sudeten. Nach verschiedenen privaten Schicksalsschlägen (ihr erster geliebter Mann stirbt an Tuberkulose, der zweite, wenig geliebte Mann nimmt sich das Leben, die Nachricht vom Soldatentod des Schwiegersohns trifft ein) sieht sich Emma den politischen Transformationsprozessen in ihrer Heimat ausgesetzt: Nach dem Einfall der Sowjets ziehen Polen in das Dorf und Emma wird mit ihrer Ziehtochter Hedwig und deren Kindern ausgewiesen. Sie gelangen nach Berlin, wo sich Hedwig das Leben nimmt. Plötzlich taucht der tot geglaubte Schwiegersohn auf und nimmt die Enkelkinder in seine Obhut. Emma findet schließlich, einem Wunder gleich, Hans Hildebrandt wieder, bei dem sie nun unterkommt.

In Borées Ein Abschied (1951) erlebt der Protagonist Marian Burger 1945 das Anrücken der Roten Armee auf Königsberg. Merkwürdig ist, dass Burger die Aus-

Folge 30 Prozent Oberschlesiens an Polen fielen. Kattowitz wurde polnisch und Hauptstadt der autonomen Provinz Schlesien sowie Sitz des schlesischen Parlaments. Vgl. http://www.deutsche-und-polen.de/orte/ ort_jsp/key=kattowitz_katowice_3.html [Stand: 18.06.2013].

${ }^{7}$ Vgl. zum Leben Ruth Storms den Beitrag von Eugeniusz Klein: Vertreibung und Verständigung im literarischen Werk von Storm, in: Schlesien - Kunst, Wissenschaft, Volkskunde, 38. Jg., H. 1, 1993, S. 43-51, in dem der Autor immer wieder Bezug auf Storms Tagebuchaufzeichnungen nimmt. Ferner http://kulturportal-west-ost.eu/biographies/storm-ruth-3/ [Stand: 18.06.2013].

${ }^{8}$ Zur besonderen Geschichte der Stadt Görlitz vgl. überblicksartig http://www.deutsche-undpolen.de/orte/ort_jsp/key=goerlitz_zgorzelec.html [Stand: 21.06.2013].

${ }^{9} \mathrm{Vgl}$. Zu Borées Biographie http://kulturportal-west-ost.eu/biographies/boree-karl-friedrich-2/ [Stand: 24.06.2013].

${ }^{10}$ Zum Leben Ruth Hoffmanns vgl. Sabine Geck: Verdrängung der Vergangenheit in der Kinderliteratur? Die Poosie-Bände von Ruth Hoffmann, in: „Erzählen müssen, um zu überwinden“. Literatura y supervivencia, hrsg. von Marisa Siguan u.a., Barcelona 2009, S. 443-455, hier i.b. S. 443f. 
nahmesituation nutzt, um seine Ehe zu beenden: Er bringt seine Ehefrau Sybille auf einem Flüchtlingsschiff unter, macht sich selbst aber auf den Weg in das nahegelegene Dorf Fischhausen, in das Haus seiner ehemaligen Verlobten Ulrike. Diese hat soeben versucht, sich zu vergiften, um einer möglichen Schändung durch die Russen zu entgehen, wurde aber durch einen Stabsarzt gerettet. Burger wacht nun an ihrem Bett, bis er, als er einen Feldgendarmen herbei reiten sieht, der ihn zum Volkssturm einziehen will, Ulrike erschießt, um sie nicht dem Kommenden anheim fallen zu lassen. Nach dieser Tat verlässt Burger Königsberg auf einem Flüchtlingsschiff, dies zwar wehmütig, doch auch mit einer gewissen Abenteuerlust.

Storms Das vorletzte Gericht (1953) spielt in der schlesischen Provinz. Marianne, die Tochter des Sägewerkbesitzers Erpach, kommt im Herbst 1944 aus Berlin zurück in ihre Heimat, um sich von ihrem im Sterben liegenden Vater zu verabschieden. Er ist der letzte Herr des Hofes, den er seiner Tochter hinterlässt. Auf diesem Hof kreuzen sich nun die Wege unterschiedlichster Gestalten, die geradezu prototypisch zu Erzählungen der letzten Kriegstage gehören: alte Nachbarn, die (wie auch Mariannes Bruder) zum Volkssturm eingezogen werden, verletzte Soldaten, die in dem Sägewerk arbeiten sollen, ausgebombte Westdeutsche, Flüchtlinge, die mit ihren Trecks von Osten nach Westen Halt auf dem Hof machen, dann sowjetische Soldaten und schließlich Polen, die das Anwesen in Besitz nehmen. Marianne harrt auf dem Hof mit einer kleinen Gruppe Deutscher aus, sieht sie es doch als ihre Pflicht an, nicht zu fliehen. Erst die administrativen Umsiedlungen im Sommer 1946 zwingen sie, die Heimat zu verlassen. Marianne gelangt in eine große Stadt im Westen, wo sie mit dem an Kindes statt angenommen Gerhart in einer Gartenlaube Zuflucht findet. Dass dies nicht ihre letzte Station sein wird, wird am Ende der Erzählung angedeutet.

\section{Erzähldramaturgie - „Anfang vom Ende“6}

Wie zu Beginn bemerkt, und wie aus den erfolgten knappen Inhaltsangaben hervorgeht, fokussieren die genannten (wie die meisten frühen Romane zum Thema) klar und kurz begrenzte Zeiträume. Die Auswahl und Einbettung der erzählten Zeitabschnitte ist von dramaturgischer Bedeutung.

Die erzählte Zeit ist besonders in Borées Ein Abschied auffällig kurz: Die Erzählung konzentriert sich ausschließlich auf die letzten Wochen Burgers in seiner Heimat. Anfangspunkt der Handlung ist der Januar 1945 und dieser Ausgangspunkt kündigt bereits vom Ende. Boreé vergleicht Königsberg im ersten Satz des Romans mit einem Todgeweihten, der ,an sein nahes Ende nicht glaubt“ (A 5). Einerseits beschreibt der Autor das Gefühl, das die deutsche Bevölkerung im Osten in den letzten Kriegsmonaten teilt: Trotz aller unverrückbaren Vorboten, trotz besseren Wissens und gegen jede Vernunft, wollte man die Hoffnung nicht aufgeben und das 
Ende ignorieren. ${ }^{11}$ Andererseits stellt Borée schon hier das Ende Königsbergs und auch das formale Ende des Romans in Aussicht.

Auch in Das vorletzte Gericht streift bereits der Titel die Frage um Anfang und Ende - es wird vom vorletzten, nicht vom letzten Gericht erzählt, das aber inhärent antizipiert wird und das der Leser unweigerlich als das Gericht Gottes am Jüngsten Tage begreift. Storm schafft also eine Analogie zwischen Endgericht und dem Verlust der schlesischen Heimat, hebt aber gleichzeitig einen gravierenden Unterschied hervor: Das göttliche Gericht wird das letzte sein und das , endgültige' Ende und die Ewigkeit markieren. Nach dem Verlust Schlesiens hingegen wird es weitergehen. Nur wie? - Mit einer Buße im „Fegefeuer“ oder mit einer Erlösung? ${ }^{12}$ Im Titel klingt also eine apokalyptische Semantik an. ${ }^{13}$ Über eine solche Semantik hinaus können sich Erzählungen einer apokalyptischen Dramaturgie bedienen. Nach Bernhard Mc Ginn ist das Gericht Teil einer apokalyptischen Matrix, die er als Trias von Krise, Gericht und Erlösung beschreibt. ${ }^{14}$ Tatsächlich schildert Storm - genau wie der Titel ankündigt - die Phase zwischen Anfang und Ende, das „Gericht“, ohne allerdings von einer vorhergehenden Krise oder gar einer Erlösung zu erzählen. Diese Erzähldramaturgie weist schon auf eine apokalyptische Deutung des Zeitgeschehens hin.

Im Roman selbst wird das Ende Schlesiens ${ }^{15}$ bereits zu Beginn motivisch aufgerufen: Marianne, die Tochter des Sägewerkbesitzers Erpach, kommt im Herbst 1944 zurück in ihre schlesische Heimat, um sich von ihrem im Sterben liegenden Vater zu verabschieden. Er ist der letzte Herr des Hofes, den er nun seiner Tochter hinterlässt. Das Schicksalsmotiv ist somit schon im ersten Kapitel aufgerufen und das Ende ist antizipiert. So lauten die letzten Worte des Vaters vor seinem Verscheiden: „Es ist vorbei, Marianne. [...] Nicht nur mit mir - mit allem - mit allem.“(VG 22). Plastisch fragt sich die zurückbleibende Tochter: „Wird die alte Seele des Hauses jetzt mitgestorben sein?" (VG 22). Wird wenige Seiten später der Geist des Vaterhauses mit dem „Geist der Heimat“ (VG 28) gleichgesetzt, so wird das Schicksalsmotiv mehr als deutlich erläutert: Das Ende des Vaters ist das Ende der schlesischen Hei-

\footnotetext{
${ }^{11}$ Vgl. etwa Thomas Darnstädt und Klaus Wiegrefe: Vater, erschieß mich!, in: Die Flucht, hrsg. von Stefan Aust u. Stephan Burgdorff, Bonn 2005, S. 21-38, hier S. $23 \mathrm{f}$.

${ }^{12}$ Mit dem Titel wird auch die Frage des Schuldiggewordenseins angerissen, die im Roman selbst nicht tangiert wird.

${ }^{13}$ Zur apokalyptischen Semantik, dem Inventar apokalyptischer Bilder, vgl. dazu Alexander K. Nagel: Ordnung im Chaos - Zur Systematik apokalyptische Deutung, in: Apokalypse. Zur Soziologie und Geschichte religiöser Krisenrhetorik, hrsg. von Alexander K. Nagel, Bernd U. Schipper, Ansgar Weymann, Frankfurt a.M./New York 2008, S. 49-72, hier besonders S. 53-58. Zu apokalyptischen Bildern in Das vorletzte Gericht siehe weiter unten.

${ }^{14}$ Vgl. Hinweis auf Mc Ginn: Apocalyptic traditions bei Nagel: Ordnung im Chaos, S. 58.

${ }^{15}$ Dieses Ende Schlesiens entspricht aber nicht dem Ende der apokalyptischen, dreistufigen Matrix, der Erlösung, sondern ist Teil der zweiten Phase: Es wird gerichtet und mit dem Verlust gebüßt. Die Erlösung steht als unbestimmte in ferner Zukunft aus.
} 
mat. Eine derartig drastische Erklärung des Bildes erfolgt erneut, wenn der alte Nachbar Neubauer sagt: „Wenn er jetzt meint [...], daß es mit allem vorbei ist, so meint er nicht nur sein Haus, uns oalle meint er, ju - uns oalle!“ (VG 31). Auffällig ist hier, dass Neubauer schlesischen Dialekt spricht, der unterstreicht, wer hier mit „uns“ gemeint ist: nämlich das schlesische Volk mit seiner Sprache, seinen Eigenarten und Traditionen, die ebenfalls einem Ende entgegen gehen.

Hoffmanns Die schlesische Barmherzigkeit wartet unter den hier versammelten Romanen mit der längsten erzählten Zeitspanne auf. Dennoch ist auch in diesem Roman die zeitliche Komposition mit dem inhaltlichen Geschehen, mit dem Anfang und Ende der schlesischen Heimat verknüpft. Die ersten zwei Drittel des Romans kommen ohne konkrete Zeitangaben aus, was diese Zeitspanne mit dem Eindruck einer unangetasteten, vor-zivilisatorischen Zeit belegt. Dieser erste Teil des Romans umfasst eine erzählte Zeit von ungefähr 50 Jahren. Das letzte Drittel des Romans beschreibt sodann die Jahre 1945/46. Somit wird ein Drittel der Erzählzeit für 1/50 der erzählten Zeit verwendet. Dem Vertreibungsgeschehen kommt also deutlich ein besonderer Stellenwert zu. Gleichzeitig bewirkt diese relative Zeitraffung im letzten Drittel eine Verstärkung der erzählten Gefühle - der Überrumpelung, der Hektik, der Verzweiflung. Die eher langsame Erzählweise in den ersten beiden Dritteln hingegen unterstreicht die Ruhe und den Frieden, der hier, einem schlesischen Attribut gleich, hervorgehoben und mit dem Einfall der Sowjets jäh unterbrochen wird. Hoffmann inszeniert also eindringlich die verlorene Vorkriegswirklichkeit - sowohl als verlorene Zeit als auch als verlorener Ort. ${ }^{16}$

\section{Zwischen Flucht und Vertreibung}

Die schlesische Barmherzigkeit fokussiert das schlesische Leben vor dem Einfall der Sowjets. Deren Einzug wird geradezu beiläufig, weder als Erlösung noch als Gefahr, erzählt: „Das große schlesische Drunter und Drüber hatte begonnen.“ (SB 245) Flucht spielt im Roman keine Rolle, dafür aber die Zeit zwischen dem Einzug der Polen und der Ausweisung der Deutschen. Von diesem Zeitraum, in dem Polen und Deutsche gemeinsam in den okkupierten Landstrichen leben mussten, erzählen Romane relativ selten. Noch deutlicher hebt Ruth Storms Roman diese Phase hervor. Dieser ist der einzige Roman der Frühphase, der das Interregnum zwischen

\footnotetext{
${ }^{16}$ Für die gesamte Fragestellung und für die hier betrachteten Texte ließe sich der Begriff des Chronotopos, wie ihn der russische Literaturwissenschaftler Michail Bachtin geprägt hat, fruchtbar machen. Nach Bachtin sind die Kategorien Raum und Zeit untrennbar miteinander verzahnt: Der zeitliche Verlauf einer Erzählung wird ebenso vom Raum bestimmt, wie umgekehrt der Raum ,,von der Zeit mit Sinn erfüllt und dimensioniert" wird (Michail M. Bachtin: Chronotopos, Frankfurt a.M. 1986, S. 7). Erzählungen über den Heimatverlust sind im Anschluss an die Feststellung, dass verlorene Zeit und verlorener Ort stets gekoppelt sind, chronotopisch.
} 
Kriegsende und Umsiedlung in den Mittelpunkt stellt. ${ }^{17}$ Doch auch in diesem Interregnum steht die Zeit nicht still, wie der Roman immer wieder plastisch durch das Symbol der Uhr verdeutlicht: Als Marianne und ihre Nächsten zunehmend erkennen, dass sie sich für oder gegen die Flucht entscheiden müssen, heißt es: „Eine Weile verging in Schweigen, nur das hastige Ticken der Küchenuhr war hörbar.“(VG 123) Die Zeit läuft - eine Entscheidung muss so schnell wie möglich gefällt werden, bald werden die Russen vor der Tür stehen. Als diese dann wenig später das Haus plündern, entwenden sie auch die alte Standuhr:

Als die alte Standuhr, die Mond und Sterne im Zifferblatt führte, herausgeschleppt wurde, lief ein Beben durch Marianne. Das Ticken der alten Uhr war der gleichmäßige Herzschlag ihres Vaterhauses gewesen, immer hatte sie ihn vernommen. [...] Der Herzschlag des alten Wohnhauses war nun für immer zum Stillstand gebracht. (VG 220)

Dieses Bild schließt die Endgültigkeit des Verlusts ein. Noch aber will die Gruppe in der Heimat verharren. Sie hat den Verlust zwar erkannt, will aber noch nicht an die endgültige Ausweisung glauben. Als nun die Polen eingezogen sind und die deutsche Bevölkerung beginnt, Hunger zu leiden, taucht nochmals das Symbol der Uhr auf: Die deutsche Gruppe tauscht immer mehr Gegenstände mit den Polen gegen Nahrung. Entsprechend lässt der kleine Gerhart eines Tages beim Mahl verlauten: „Heute essen wir die Uhr auf, vielleicht habe ich den kleinen Zeiger auf dem Brot und die Mutter den großen und die Tante das Zifferblatt und der Großvater das Gehäuse“(VG 259). Nun ist, wie der Erzähler dann auch unmissverständlich erklärt, das Ende gekommen:

Allen blieb der Bissen im Halse stecken, und es war ihnen klar, der letzte Akt hatte begonnen; wenn die Substanz einer Familie aufgezehrt wird, um den Lebensunterhalt zu ermöglichen, so ist das ein untrügliches Zeichen für das nahe Ende. $(\text { VG } 259)^{18}$

\footnotetext{
${ }^{17}$ Im Roman wird dieser Fokus auf die Zwischenzeit bzw. die Transformation auch immer wieder deutlich angesprochen. Etwa: „Wir leben in dieser Übergangszeit und werden von den Gegensätzen hinund hergerissen [...]“ (VG 209), „Übergangsmenschen“ (VG 213) oder ,[...] vielleicht war auch eine Neugier dabei, wie sich dieser Zwischenzustand einmal auflösen möge“ (VG 235).

${ }^{18}$ Śliwińska nennt weitere Beispiele von Flucht- und Vertreibungstexten, in denen Uhren den „Bruch im Kontinuum der biographischen und historischen Zeit“ symbolisieren, etwa Horst Bieneks Erde und Feuer von 1982 und Grete Scholz-Gauers Familienchronik aus Schlesiens schwerster Zeit (so der Untertitel) Gejagtes Volk. Katarzyna Śliwińska: Eine deutsche Odyssee? Figurationen der Irrfahrt in der deutschen Literatur über Flucht und Vertreibung, in: Linguae Mundi, 5, Poznań 2010, 127-150, hier S. 141. Auch im Film Auf einem langen Weg symbolisiert die Zerstörung einer alten deutschen Standuhr durch die Russen das Ende.

Neben der Uhrensymbolik greifen Texte zum Thema auch oft Wendung aus dem semantischen Feld „Theater“ auf, hier „,der letzte Akt“, womit den Vorgängen, gar dem Weltgeschehen eine deutliche Chronologie, ja geradezu ein Handlungsmuster zugeschrieben wird (besonders auffällig in Werner Kloses Jenseits der Schleuse, Tübingen 1953).
} 


\section{Ankunft im Westen - Der Neubeginn?}

Wie eingangs erwähnt, schildern nur wenige frühe Romane das Eintreffen im Westen. Geschieht dies, bleibt fraglich, ob die Ankunft als ein Neubeginn aufgefasst bzw. dargestellt wird.

Die schlesische Barmherzigkeit beschreibt die Ankunft der Protagonistin Emma im Westen, dort zunächst in einem Vertriebenenlager und schließlich in einem ,neuen Zuhause', das für Emma der wiedergefundene Hans Hildebrandt ist. Folglich ist das neue Zuhause letztlich das alte Zuhause. Damit wird der Inklusionsmechanismus der Vertriebenen angesprochen:

Er [der Flüchtling] ist ein Isolierter, den keiner kennt, und der keinen kennt. Von großer Wichtigkeit für den Entwurzelten ist daher, sein Landsmann'. Wo immer er ihm begegnet, trifft er den Vertrauten, der die gleiche Sprache redet und ,Bescheid weiß`, sein Bruder im Flüchtlingsleide ist, für den er nicht der Fremdling und Ausgestoßene ist. ${ }^{19}$

Das Neue ist kein vollständig Neues, sondern ein Neues im Alten. So stellt der Roman die Ambivalenz, die dem Neubeginn für die Flüchtlinge im Westen notwendig zugrunde liegt, dar und markiert diese wiederum durch den erzählerischen Umgang mit Zeit: Am Ende der Erzählung, im Kapitel „Dach über dem Kopf“, steht die Ankunft Emmas in dem neuen alten Zuhause. Der Text bezweifelt somit die Integration der Vertriebenen im Westen.

Typisch für Romane zum Thema (wie übrigens auch für andere Romane, die von globalen Katastrophen erzählen) ist zudem, dass die formalen Kategorien des Textes ,Anfang' und ,Ende' eben aufgrund des verhandelten Themas in den Diskurs einbezogen und semantisiert werden. ${ }^{20}$ Solche Texte erzählen zu Beginn oft ein Ende und am Ende postulieren sie in der Regel einen Neubeginn. Im formalen Abschluss der Narration muss also ideologisch ein Beginn aufleuchten:

Dieser wird zumeist durch die Macht der Wörter gestützt, insofern die letzten Sätze und Wörter der Texte dem Wortfeld des Beginns, des Anfangs entnommen sind und/oder unter das Paradigma der Offenheit subsumiert werden können. ${ }^{21}$

Das trifft auffällig auf Das vorletzte Gericht zu. Zum einen sieht die Protagonistin ihre persönliche zukünftige Aufgabe darin, dem ,heimatlosen Jungen [Gerhart] Heimat zu sein" (VG 350), zum anderen sieht sie sich für einen allgemeineren Dienst am deutschen Volk bestimmt. Die Gartenlaube, in der sie mit dem Jungen zunächst im Westen untergekommen ist, erhält nun den Stempel eines Zwischenspiels, als ihr ein alter Vertriebener, wohlgemerkt auf der vorletzten Seite des Textes, sagt:

\footnotetext{
${ }^{19}$ Elisabeth Pfeil: Der Flüchtling. Gestalt einer Zeitwende, Hamburg 1948, S. 61.

${ }^{20}$ Vgl. Hans Krah: Weltuntergangsszenarien und Zukunftsentwürfe: Narrationen vom „Ende“ in Literatur und Film 1945-1990, Kiel 2004, S. 353.

${ }^{21}$ Ebd. S. 353. Das „Paradigma“ der Offenheit bedient das Ende von Borées Ein Abschied.
} 
Sie haben sich hier etwas Ehrliches aufgebaut, um den Übergang zu ertragen - aber auf die Dauer kann das nicht Ihr letztes Ziel sein. Wir müssen uns geistig wach halten. [...] [W]ir glauben, [...] daß Sie der verlorenen Erde und ihrem Sohn dadurch am besten dienen würden, wenn Sie die Heimat geistig miterhalten helfen. (VG 353)

Sodann schenkt er Marianne ein Büchlein, das ,anregen soll, all das festzuhalten“(VG 353), was sie erlebte. Das Büchlein enthält leere Blätter - also den Aufruf, das Erlebte für die Zukunft in einer niedergeschriebenen Erzählung zu archivieren. Auf der ersten Seite des fiktiven Büchleins steht ein Gedicht, das auf der letzten Seite des Romans abgedruckt ist, und das eben Formulierungen enthält, die an das formale Ende des Textes einen ideologischen Anfang setzen:

Er [der goldene Stern der Seelenschätze] wird zu neuem Glanz aufsteigen / [...] / nur werden so den Tod wir / und die Armut überwinden / bis einst uns unsere Kinder / Frühlingskränze um die / versorgten Stirnen binden. (VG 355)

Marianne soll nun die Aufgabe der Chronistin für „gleichgesinnte[] Menschen“ (VG 353) übernehmen. Damit inszeniert Storm ihren eigenen Roman als dieses Erinnerungsdokument und sie selbst erhebt sich zur Sprecherin des Lesepublikums. Denkt man hier die im Roman angelegte apokalyptische Idee konsequent zu Ende, wird zwar deutlich, dass die dritte Stufe der Trias ,Krise - Gericht - Erlösung' noch nicht erreicht, man ihr aber doch näher gekommen ist. Hier offenbart sich die Wirkungsabsicht der apokalyptischen Erzählweise: das Bemühen, aufzurütteln und zur Agitation zu bewegen (,apokalyptischer Aktivismus"). Standen zwar weite Teile der Erzählung noch unter dem Stern des Erduldens (,,apokalyptischer Quietismus“) und fungiert der Roman durch die Schilderung einer (mythisch verklärten) Vergangenheit auch als Trostschrift, so ergehen nun Anweisungen zur Verwirklichung des „Heils“, der „Erlösung“ ${ }^{22}$ Marianne geht dem Leser dabei als leuchtendes Beispiel voran.

\section{„Was nennen Sie außerdem den Anfang? 1933 - die Aufrüstung - oder Stalingrad?““}

Wo nun liegt der Anfang dieser Geschichte, der Geschichte vom Heimatverlust? Gibt es Hinweise auf das, was Flucht und Vertreibung vorausging? Wird der Transformationsprozess in den Ostprovinzen als Teil eines größeren Prozesses, gar als Folge eines anderen Ursprungs - der Gräueltaten der Nazis - gesehen?

Diese Verbindung ruft Borée sehr früh auf. Es heißt:

„Sie wissen ganz gut, was ich meine. - Ich habe die Geschichte von Anfang an verflucht.“ „Aber nicht sehr vernehmlich. Was nennen Sie außerdem den Anfang? 1933 - die Aufrüstung - oder Stalingrad?“(A 56)

\footnotetext{
${ }^{22}$ Vgl. Nagel: Ordnung im Chaos, S. 62-67.
} 
und

Seit Stalingrad reifte das, was er für seine Person vom ersten Kriegstage an hatte wachsen sehen, das Schicksal dieser Stadt, das Schicksal der Provinz: Dieser Krieg würde von Deutschland mit Ostpreußen bezahlt. (A 11)

Schuld ist Deutschland, Schuld ist nicht das Schicksal, oder ein teleologischer, apokalyptischer Geschichtsverlauf, wie ihn viele andere Texte der Zeit antizipieren. Unternimmt der Protagonist Burger zwar den Versuch, die vorhergehende Katastrophe mit einer historischen zu vergleichen, erkennt er doch, dass das Auffinden eines äquivalenten Vergleichsmoments die Schuld der Deutschen verwischte. Er sinniert:

Die Geschichte versah sich um drei Jahrtausende, sie war zurückgefallen in abgelegte, barbarische Gebräuche. Burger lächelte: Die Geschichte? - Nein, der Mensch, das liebe deutsche Volk, das jetzt erwachte. (A 14)

Burger ruft nicht nur den Ursprung von Flucht und Vertreibung in Erinnerung, sondern weist auch darauf hin, dass das Schicksal der Ostprovinzen kein exklusives ist. Opfer waren und bleiben die von Deutschland angegriffenen Völker: „Wann werden unsere treuen Mitbürger wohl beginnen, sich an Warschau oder an unseren Einmarsch in Frankreich zu erinnern?" (A 15) Hier wird ein typisches Verfahren westdeutscher Nachkriegsprosa deutlich: Reflexionen über die Vergangenheit, auch über ihre Ursachen, ergehen in erster Linie auf der Ebene der Figurenrede, teilweise auch durch die Erzählinstanz, selten aber spiegelt sich die Reflexion in den narrativen Modi selbst. Ästhetische Experimente kommen in der Frühphase der Literatur zum Thema wenig bis gar nicht vor (die Forschung deutet Jens Rehns Feuer im Schnee immer wieder als einzigen Gegenpol ${ }^{23}$ ), erst recht nicht solche, die durch Erzählverläufe oder -muster die Deutung des Zeitgeschehens abbildeten oder doppelten. $^{24}$

Was diese Beobachtung zu den Erzählverläufen angeht, kann nach den hier erfolgten Betrachtungen allerdings genauer nachgehakt werden: Dass etwa Hoffmanns Text zunächst von der Zeit vor der Flucht erzählt, die Reichspogromnacht (im Kapitel „Brand im Tale) und Auschwitz (etwa SB 227, 233) in dem Text Erwähnung finden, zeugt von Ursachenforschung, die auch im Erzählverlauf zum Ausdruck kommt (auch wenn diese reflektierte Kontextualisierung sicherlich eher eine Ausnahme bleibt).

\footnotetext{
${ }^{23}$ Siehe z.B. die Interpretation von Monika Melchert. Laut Melchert stimmten hier Erzählstruktur und Struktur des Mitzuteilenden überein. Vgl. Melchert: Die Zeitgeschichtsprosa nach 1945 im Kontext der Schuldfrage, S. 157.

${ }^{24} \mathrm{Zu}$ diesem Gedanken vgl. Mathias Bertram: Literarische Epochendiagnosen der Nachkriegszeit, in: Deutsche Erinnerung. Berliner Beiträge zur Prosa der Nachkriegsjahre (1945-1960), hrsg. von Ursula Heukenkamp, Berlin 2000, S. 11-100, hier i.b. S. 11f.
} 


\section{Rückkehr? Revanchismus?}

Die schlesische Barmherzigkeit zeugt nicht nur von der humanistischen, sondern auch realistischen Sicht der Autorin. Revanchismus, der Anspruch der Vertriebenen auf die Ostgebiete, wird im Roman in keiner Weise ausgedrückt. Das Gegenteil ist der Fall: Die Einsicht, dass die Heimat endgültig verloren ist, steht im Vordergrund, ohne dass dabei Vorwürfe oder Besitzansprüche geltend gemacht würden. Vielmehr spricht aus der Erkenntnis des Verlusts die Einsicht der Schuld, aber sie ist auch Grund zu privater Trauer. „Nie wieder“ - diese Worte stehen fast programmatisch im Zentrum des Kapitels „Der geopferte Wald“. Die Formulierung leitet jede Aussage ein, die beschreibt, welche sinnlichen Erfahrungen die Protagonistin aufgrund des Heimatverlusts nie wieder machen wird. Diese Anapher drückt die Trauer über den intimen Verlust aus - nie wieder wird Emma den Pilzhauch spüren, nie wieder den Nussgeruch: „,[D]ie Luft ist voll vom Niewieder, dein Herz zerbricht von seiner Hundertfältigkeit, dein Ohr ertaubt vor seiner Klage, dein Auge weint jeder Farbe nach [...].“ (SB 265) ${ }^{25}$

Auch der Titel des Romans Ein Abschied zeigt, dass ein endgültiges Ende in seinem Zentrum steht. Findet der wirkliche Abschied von der Heimat zwar erst am Ende der Handlung statt, so ist doch die gesamte Erzählung als Abschied konzipiert. Es wird weniger ein Übergang als vielmehr der Prozess eines Endes erzählt. Ende ist demnach kein Punkt, sondern ein Narrativ an sich, das wiederum Anfang und Ende hat. So beginnt der Roman, wie angedeutet, mit dem sprichwörtlichen „Anfang vom Ende“. Durch den Vergleich der Stadt mit einem Todgeweihten erhält aber auch der Titel Abschied einen anderen Charakter: Es ist ein endgültiger Abschied, es wird keine Wiederkehr geben. Pfeils Behauptung

Auch in ihren [der Flüchtlinge] Weggang ist eine Hoffnung eingewirkt, wenn auch eine andere, rückwärts gewendete, sie gehen, um wiederzukehren. Zur Vorstellungswelt der Fliehenden gehört die Heimkehr. Sie verlassen die Heimat, ohne sie aufzugeben. ${ }^{26}$

trifft hier nicht zu. Auch Burgers Ehefrau Sybille erkennt, dass ihr altes Königsberger Leben vorbei ist und ein neues, anderes bevorsteht. Sie erklärt: „Ich habe kein Talent, mein Leben abzubrechen und zum zweitenmal zu beginnen.“(A 32) Die zur Flucht Genötigte verspürt Angst und Unmut im Hinblick auf die Zukunft. Sie nimmt den Aufbruch als $A b$ bruch wahr und das Ziel der Fluchtbewegung im Westen als einen Punkt, an dem nicht weitergemacht werden kann, sondern neu begonnen werden muss. Genau genommen wird also kein Übergang, keine Transformation im Sinne eines kontinuierlichen Wandels aufgerufen, sondern ein Umbruch, ein abgebrochener Lebenslauf gar, ein Leben, das zum zweiten Mal im Ungewissen gestartet werden muss.

\footnotetext{
${ }^{25}$ Ruth Storm formuliert ähnlich: „das Land Nimmermehr“ (VG 217).

${ }^{26}$ Pfeil: Der Flüchtling, S. 13.
} 
Die Beziehung zwischen Sybille und Marian Burger wird als pragmatische beschrieben, die Beziehung zu Ulrike als fortwährende Liebe. Beide Trennungen stehen symbolisch für die Trennung von der Heimat. Auf der einen Seite muss Burger sich schmerzvoll von seiner geliebten Heimat trennen, diese begraben. Auf der anderen Seite löst er sich nicht nur egoistisch, sondern pragmatisch und abenteuerlustig von Sybille. So fühlt er sich beim Verlassen der Heimat tatsächlich frei und gesegnet:

Aber das Ende, das er jener Frau auf dem Gutshof hatte bereiten müssen, erschien im jetzt wie das bittere und doch versöhnliche Begräbnis, das er dem Lande und dem Leben, die er beide verließ, selber habe zurichten dürfen. (A 206)

Es wird sein persönlicher, aktiver Abschied unterstrichen, der ihn frei macht. Dabei geht es nicht um den Abschied von Ostpreußen - ,ihm verlangte nicht einmal danach" (A 208), sondern um seine ganz persönlich, ja egoistische Freiheit, die er auf dem Schiff in einer zugewiesenen Hängematte als ,physische Empfindung aufgehobener Schwere mit dem Bewußtsein, daß er davongekommen und frei sei“ (A 207) empfindet. Es wird so auch ein offener, durchaus abenteuerfreudiger Blick in eine unbestimmte Zukunft angedeutet. Liegt diese Zukunft zwar im Ungewissen, und wird dann auch nicht erzählt, wird sie dennoch nicht als eine negative, bedrohliche antizipiert. Burger kehrt, trotz der Tränen, die er bei seiner Abfahrt weint, nicht in trauriger Verzweiflung seiner Heimatstadt den Rücken.

\section{Der Blick in die Zukunft: Sinnstiftungen durch Geschichtsauffassung}

Den Verlust Königsbergs hat Burger auf sich zukommen sehen und er interpretiert ihn als logische Konsequenz der Kriegsgräuel der Nationalsozialisten. Diese Erkenntnis seines Protagonisten geht mit Borées eigener Auffassung einher: Für Borée, so Arno Lubos, hat der Mensch sein Schicksal selbst in der Hand. ${ }^{27}$ Auch in diesem Roman macht der Autor also nicht den Lauf der Welt für die Erfahrungen des Einzelnen verantwortlich.

Weitaus typischere erzählerische Versuche, das Geschehen zu deuten, unternimmt Storm. Sie konterkariert Bilder vom Ende häufig mit Bildern des Anfangs, etwa im ersten Teil des Romans: Ein Lastkraftwagen der Wehrmacht fällt versehentlich die große Blautanne vor dem Sägewerk, die als Wahrzeichen des Hofes gilt. Der obere Teil der Tanne findet am letzten Kriegsweihnachtsfest 1944 seinen Platz in der Wohnstube und wird von oben bis unten mit zahlreichen Kerzen und anderem Schmuck besetzt:

${ }^{27}$ Vgl. Arno Lubos: Geschichte der Literatur Schlesiens, Bd. 3, München 1974, S. 373. 
Waren nicht all die bunten Kindheitsdinge auf dem Baum in dem schemenhaften Licht der einen Kerze, wie ein langsames Verlöschen einer Zeit, die niemals wiederkehrte? War nicht alles um sie herum schon unwirklich, und waren nicht schon Zeichen da für eine neue Verkündung? Waren nicht die Mutter aus dem Rheinland und die Soldaten nicht schon losgelöst aus dem Ring der alten Ordnung? Warteten sie nicht unbewußt auf eine Erlösung, auf einen neuen Stern der Verheißung? (VG 89)

Hier wird deutlich von einem endgültigen Ende gesprochen und gleichzeitig in die Zukunft geblickt. Dass der Leser den Fall der Tanne motivisch und als die folgenden historischen Ereignisse ankündigend verstehen soll, garantiert der Erzähler durch eine geradezu aufdringliche Unterstreichung des Bildes: „War der Baum nicht ein Symbol? Er war wohl durch rohen äußeren Eingriff gestürzt, aber hier im Weihnachtszimmer hatte er Auferstehung gefeiert zur Freude aller." (VG 90). Wiederum wird implizit ein Blick in die Zukunft gerichtet, der sich auffällig der christlichen Heilsgeschichte bedient: Die Rede von einer Auferstehung antizipiert Erlösung nach Krise und Gericht und verweist auf die Idee der Sündenvergebung. Tatsächlich projiziert Storm, wie Helbig erkennt, immer wieder den Urzustand in die Zukunft. ${ }^{28}$ Sie deutet, ohne dass dieser Ausdruck fiele, die Vorgänge gleich der Sintflut, nach der die Menschen die Möglichkeit zum Neuaufbau und zum endgültigen Gotterkennen erhalten:

Gottes Schöpfung wurde verstümmelt, entstellt und entweiht, vielleicht lag der einzige Sinn darin, $\mathrm{da} ß$ der empfindsame Mensch diese Bilder nie mehr vergaß, beständig das höchste Ziel der Menschheit, das Ziel des Friedens und der Verständigung unter den Völkern anzustreben, dann - ja dann wäre auch das Leiden der hilflosen Geschöpfe nicht umsonst gewesen. (VG 121)

Solche prophetischen Sinngebungsversuche finden sich häufig in den Nachkriegsromanen und weisen auf ein teleologisches Geschichtsbild hin: Ob als Sintflut oder Apokalypse gedeutet, immer wird die Katastrophe als notwendiges, kathartisches Element ausgelegt, dem der paradiesische Urzustand vorausging und der erst nach und durch ihre Bewältigung wieder erreicht werden kann.

Auch Hoffmanns Die schlesische Barmherzigkeit ruft diesen Urzustand bzw. die Läuterung in der Zukunft auf. Die letzten Sätze des Textes beschreiben ein Gebet Emmas und antizipieren damit eine weitere Heimstatt, eine endgültige. Dennoch eben diesem Endgültigen verweigert sich der Roman. Er schließt mit den Worten:

Wann der endgültige Schlaf sie zu sich nahm oder nehmen wird und einbetten, will einer von uns das wissen? Sind wir nicht dessen sicher, daß sie kommt und geht, solange Erbarmen seine Boten ausschickt, zu den Kindern, damit sie nicht verderben, zu Mann und Weib in der strengen Zeit, zu den Verlassenen, zu den Alten, zu dir, zu mir? (SB 329)

Das Spiel mit dem Tempus fällt auf: „Zu sich nahm oder nehmen wird“ - von welchem Zeitpunkt aus wird erzählt? Das Tempusspiel, die Redewendung des Kommens und Gehens und der Ausdruck „solange“ rufen nun ein Überzeitliches auf

\footnotetext{
${ }^{28}$ Vgl. Louis Ferdinand Helbig: Der ungeheure Verlust. Flucht und Vertreibung in der deutschsprachigen Belletristik der Nachkriegszeit, Wiesbaden 1996, S. 121.
} 
und Emmas Geschichte gerät damit sowie durch das plurale „wir“ zur Geschichte des Lesers. Ein im Roman oft impliziertes teleologisches Geschichtsverständnis scheint auch hier auf und entspricht der allgemeinen, zeitgenössischen Interpretation des historischen Geschehens auf der Suche nach Sinngebung. Solche Deutungen sind etwa auch im wissenschaftlichen Diskurs virulent; Elisabeth Pfeil interpretiert in ihrer soziologischen Studie: „Im Grunde sind es die ewigen Erfahrungen der Menschheit, die der Flüchtling macht, die uralten Wahrheiten kommen wieder zu Ehren. ${ }^{\prime 29}$

Die Romane experimentieren, wie angedeutet, in der Regel nicht erzählerisch mit der Zeit; vielmehr verfolgen die meisten Romane ein klassisches chronologisches Erzählmuster. ${ }^{30}$ Auch das chronologische Erzählen ist Abbild der vorherrschenden Sinnstiftungsversuche:

Die Finalität als Organisationsprinzip des Erzählten wird [...] in das Erzählte hinein projiziert, die zum historischen Verlauf geordnete Kontingenz wird dadurch mit einer Tiefendimension ausgestattet, in der mehr oder minder personal gedachte „Mächte“ wirken. ${ }^{31}$

Śliwińska nennt diese finale Orientierung eine Viktimisierungsstrategie. ${ }^{32}$ Werden nämlich die letzten Tage in der Heimat und damit der Einfall der Sowjets in dieselbe beschrieben, dann wird vorwiegend von den Brutalitäten der Russen erzählt, nicht aber von den vorhergehenden deutschen Gräueltaten. Mit einer solchen zeitlichen Fokussierung tun die Texte geradezu so, als habe es die deutsche Täterschaft gar nicht gegeben, erwähnen sie diese doch mit keinem Wort.

\section{Narrationen einer ,Zeitenwende“? - Fazit und Ausblick}

„Die Gewichte verschieben sich unter dem Druck der losgelösten und in Bewegung geratenen Massen, ,die alten Gefüge zerfallen und neue wollen sich bilden. ““33 So schreibt die Soziologin Elisabeth Pfeil 1948 in einer der ersten Studien zum Thema Flucht und Vertreibung. Den Flüchtling, den Titelgeber ihrer Betrachtung, nennt sie im Untertitel „Gestalt einer Zeitenwende“.

Frühe Romane zum Thema empfinden, wie gezeigt, diese Zeitenwende und markieren sie erzählerisch. Neben der zum Tragen kommenden Zeitsymbolik gibt vor allem die Erzähldramaturgie Auskunft über die Deutung des Zeitgeschehens. In

\footnotetext{
${ }^{29}$ Pfeil: Der Flüchtling, S. 217.

${ }^{30}$ Womit wohl auch ein Hinweis auf das intendierte Lesepublikum ergeht: Es wird für die breite Masse und weniger für literarisch geübtes und anspruchsvolles Publikum geschrieben.

${ }^{31}$ Katarzyna Śliwińska: Eine deutsche Odyssee?, S. 136. Diese Interpretation Śliwińskas zu Werner Kloses Jenseits der Schleuse lässt sich auf die untersuchten und andere Nachkriegsromane ausweiten.

${ }^{32}$ Vgl. ebd., S. 135.

${ }^{33}$ Pfeil: Der Flüchtling, S. 6.
} 
der Regel liegen den Texten apokalyptische Sinnstiftungsversuche zugrunde, die sich in Titeln, Figurenrede oder im Erzählverlauf spiegeln. Dabei bleibt der erzählerische Umgang mit der Zeit aber ein klassisch-gewöhnlicher. Experimentiert wird wenig, was dem Leser die Identifikation erleichtert und so Trost und Sinn stiftet. Die Kategorien Anfang und Ende sind für die Texte insofern zentral, als dass sie letztlich nicht einen Übergang, sondern ein Ende erzählen. Das Ende aber wird nicht als Punkt, sondern als Narrativ (als Prozess) des Endes dargestellt. Dieses prozessuale Ende muss folglich einen Anfang besitzen, der aber nicht immer deutlich hervorgehoben wird. Das Ende des Endes bleibt hingegen immer ausgespart; der Prozess ist stets um sein Ende - im Sinne einer Erlösung - kupiert. Diese Beschneidung ist dem Thema der Vertreibung geschuldet, ja inhärent, und führt an den Anfang des vorliegenden Beitrags zurück: Die Texte erzählen den Übergang nicht zu Ende, weil er für die Autoren Anfang der 1950er Jahre nicht zu Ende ist.

Ob spätere Erzählungen des ostdeutschen Heimatverlusts den Übergang anders erzählen, sie Anfang und Ende abweichend der analysierten frühen Erzähldramaturgie setzen oder bearbeiten, ist eine richtungweisende Frage für weitere Forschungen. Sollten sich hier divergierende Beobachtungen einstellen, muss die Frage anschließen, ob ein anderer erzählerischer Umgang von einem anderen sozio-psychologischen Umgang mit dem Verlust und dem Neubeginn zeugt, ob sich also eine Bewältigung des Übergangs eingestellt hat und dieser nunmehr erzählt werden kann.

\section{Literatur}

Bachtin, Michail M.: Chronotopos, Frankfurt a.M. 1986.

Bertram, Mathias: Literarische Epochendiagnosen der Nachkriegszeit, in: Deutsche Erinnerung. Berliner Beiträge zur Prosa der Nachkriegsjahre (1945-1960), hrsg. von Ursula Heukenkamp, Berlin 2000, S. 11-100.

Borée, Karl Friedrich: Ein Abschied, Wiesbaden 1951.

Darnstädt, Thomas; Wiegrefe, Klaus: Vater, erschieß mich!, in: Die Flucht, hrsg. von Stefan Aust u. Stephan Burgdorff, Bonn 2005, S. 21-38.

Frenzel, Karolina u.a.: Storytelling: Das Praxisbuch, München/Wien 2006.

Geck, Sabine: Verdrängung der Vergangenheit in der Kinderliteratur? Die Poosie-Bände von Ruth Hoffmann, in: „Erzählen müssen, um zu überwinden“. Literatura y supervivencia, hrsg. von Marisa Siguan u.a., Barcelona 2009, S. 443-455.

Helbig, Louis Ferdinand: Der ungeheure Verlust. Flucht und Vertreibung in der deutschsprachigen Belletristik der Nachkriegszeit, Wiesbaden 1996.

Hoffmann, Ruth: Die schlesische Barmherzigkeit, Köln 1953.

Klin, Eugeniusz: Vertreibung und Verständigung im literarischen Werk von Storm, in: Schlesien Kunst, Wissenschaft, Volkskunde, 38. Jg., H. 1, 1993, S. 43-51.

Krah, Hans: Weltuntergangsszenarien und Zukunftsentwürfe: Narrationen vom „Ende“ in Literatur und Film 1945-1990, Kiel 2004

Lubos, Arno: Geschichte der Literatur Schlesiens, Bd. 3, München 1974. 
Melchert, Monika: Die Zeitgeschichtsprosa nach 1945 im Kontext der Schuldfrage, in: Deutsche Erinnerung. Berliner Beiträge zur Prosa der Nachkriegsjahre (1945-1960), hrsg. von Ursula Heukenkamp, Berlin 2000, S. 101-166.

Nagel, Alexander K.: Ordnung im Chaos - Zur Systematik apokalyptischer Deutung, in: Apokalypse. Zur Soziologie und Geschichte religiöser Krisenrhetorik, hrsg. von Alexander K. Nagel, Bernd U. Schipper, Ansgar Weymann, Frankfurt a.M./New York 2008.

Pfeil, Elisabeth: Der Flüchtling. Gestalt einer Zeitwende, Hamburg 1948.

Śliwińska, Katarzyna: Eine deutsche Odyssee? Figurationen der Irrfahrt in der deutschen Literatur über Flucht und Vertreibung, in: Linguae Mundi, 5, Poznań 2010, S. 127-150.

Storm, Ruth: Das vorletzte Gericht, München 1953.

\section{Internetquellen:}

www. kulturportal-west-ost.eu/biographies/boree-karl-friedrich-2/ (Stand: 24.06.2013).

www. kulturportal-west-ost.eu/biographies/storm-ruth-3/ (Stand: 18.06.2013).

www.deutsche-und-polen.de/orte/ort_jsp/key=goerlitz_zgorzelec.html (Stand: 21.06.2013).

www.deutsche-und-polen.de/orte/ort_jsp/key=kattowitz_katowice_3.html (Stand: 18.06.2013). 